\title{
What is known about the effects of exercise or training to reduce skeletal muscle impairments of patients with myotonic dystrophy type 1? A scoping review
}

Marie-Pier Roussel ${ }^{1,2,3}$, Marika Morin ${ }^{1}$, Cynthia Gagnon ${ }^{2,3,4}$ and Elise Duchesne $e^{1,2,3^{*}}$ (D)

\begin{abstract}
Background: Myotonic dystrophy type 1 (DM1) is a neuromuscular disease characterized by multisystemic involvements including a progressive loss of maximal muscle strength and muscle wasting. Poor lower-limb strength is an important factor explaining disrupted social participation of affected individuals. This review aims to map what is known about the effects of exercise and training programs undertaken to counteract skeletal muscle impairments in DM1 patients.

Methods: Medline, CINAHL and EMBASE databases were searched. Regarding study eligibility, title and abstract of 704 studies followed by 45 full articles were reviewed according to the following eligibility criteria. Inclusion: (1) humans with DM1 and (2) experimental protocol relying on exercise or training. Exclusion: (1) studies that do not evaluate skeletal muscle responses or adaptations, (2) reviews covering articles already included and (3) pharmacological intervention at the same time of exercise or training program.

Results: Twenty-one papers were selected for in-depth analysis. Different exercise or training protocols were found including: acute exercise, neuromuscular electric stimulation, strength training, aerobic training, balance training and multiple rehabilitation interventions. Seven studies reported clinical measurements only, five physiological parameters only and nine both types.

Conclusion: This scoping review offers a complete summary of the current scientific literature on the effect of exercise and training in DM1 and a framework for future studies based on the concomitant evaluation of the several outcomes in present literature. Although there were a good number of studies focusing on clinical measurements, heterogeneity between studies does not allow to identify what are the adequate training parameters to obtain exercise or training-induced positive impacts on muscle function. Scientific literature is even more scarce regarding physiological parameters, where much more research is needed to understand the underlying mechanisms of exercise response in DM1.
\end{abstract}

Keywords: Myotonic dystrophy type 1, Exercise, Training program, Rehabilitation interventions, Muscle weakness, Muscle atrophy

\footnotetext{
* Correspondence: elise1_duchesne@uqac.ca

'Département des sciences de la santé, physiothérapie, Université du Québec à Chicoutimi, 555, boulevard de l'Université, Chicoutimi, Quebec G7H 2B1, Canada

${ }^{2}$ Groupe de recherche interdisciplinaire sur les maladies neuromusculaires, Centre intégré universitaire de santé et de services sociaux du SaguenayLac-St-Jean, 2230 rue de I'Hôpital, Saguenay, Québec, Canada

Full list of author information is available at the end of the article
}

(c) The Author(s). 2019 Open Access This article is distributed under the terms of the Creative Commons Attribution 4.0 International License (http://creativecommons.org/licenses/by/4.0/) which permits unrestricted use, distribution, and reproduction in any medium, provided you give appropriate credit to the original author(s) and the source, provide a link to the Creative Commons license, and indicate if changes were made. The Creative Commons Public Domain Dedication waiver (http://creativecommons.org/publicdomain/zero/1.0/) applies to the data made available in this article, unless otherwise stated. 


\section{Background}

Myotonic dystrophy type 1 (DM1) is an autosomal dominant degenerative neuromuscular disease [1-3] caused by a nucleotide triplet (CTG) repeat expansion within the 3' untranslated region of the Dystrophy Myotonic Protein Kinase (DMPK) gene [4]. The discovery of the defective gene was made in 1992, which subsequently allowed a more accurate diagnosis [4]. CTG repeat length is usually moderately correlated with earlier disease onset and more severe symptoms [5]. There are four to five recognized phenotypes: congenital, infantile/ childhood, juvenile, classical/adult and late-onset [5].

DM1 is a multisystemic disease that affects skeletal muscles and cardiac, digestive, nervous and endocrine systems, to name a few. Typical signs and symptoms include myotonia, heart arrhythmias, cataracts, daytime sleepiness, cognitive impairments, apathy and muscle wasting and weakness. The latter is a major concern for affected people, which can lose up to 30.3 to $54.5 \%$ of maximum strength on a 9 year period with a progression pattern from distal to proximal muscles [6]. A recent study has shown that even DM1 patients classified in the first two grades of Muscle Impairment Rating Scale (MIRS) [7], which are considered to have no limb muscles weakness, show a 11.3 to $24.1 \%$ of maximal strength loss in lower limb muscle groups compared to predicted values [8]. Muscle weakness is a major culprit for restrictions in activities of daily living and social roles [9].

Although the physiological mechanisms are not yet fully understood, one of the main mechanisms linking the CTG repetitions to the symptoms in DM1 is the accumulation of toxic CUG RNA causing splicing difficulties by sequestrating RNA-binding proteins such as muscle blindlike-1 (MBNL-1) [10]. Originating from this, many physiological impairments have been identified in skeletal muscle affected by DM1 that could contribute to muscle weakness. At a histological level, preferential atrophy of type I myofibers, higher proportion of centrally nucleated fibers, fibrosis and fat infiltration have been reported [11]. Moreover, level of expression of glycogen synthase kinase 3 beta (GSK3 $\beta$ ), a negative regulator of muscle protein synthesis, is increased [12]. Thereafter, myogenic capacity is also perturbed as demonstrated by decreased proliferative capacity and premature senescence of satellite cells (muscle stem cells) and delayed fusion and differentiation of myoblasts (activated satellite cells) [13-17]. An increase in protein degradation and a decrease in protein synthesis $[1,18-20]$, as well as perturbed local and systemic inflammatory statuses [21, 22], have also been reported. Finally, a mouse model study has also shown that calcineurin is overexpressed in DM1, arguing that it may be a compensatory mechanism due to its essential role in muscle hypertrophy [23]. This study also showed that symptoms worsened in affected mice where calcineurin was downregulated [23]. These findings only present an incomplete view of muscle physiology in DM1, and knowledge about immediate responses and long-term adaptations to exercise or training are essential for a complete understanding.

One of the main strategies to counter maximal muscle strength loss and muscle wasting is through exercise and training. However, to be effective, exercise and training need to be specifically adapted to a population and based on an appropriate dosage. Furthermore, a rationale based on physiological knowledge is a key point to properly adapt training to populations with a specific pathology. In DM1 population, while the underlying mechanism remain unclear given the small number of studies along with the small number subjects included in these, training-induced positive skeletal muscle adaptation seems possible in DM1. Indeed, it has been shown that training can induce increase in myofiber cross-sectional area without causing any histopathological abnormalities [3, 24]. These results suggest that, in addition to the positive clinical impact of exercise in DM1 such as muscle strength gains, exercise should physiologically work in this disease. A systematic review has shown strength training to be safe [25], but has not concluded about its effect on skeletal muscle impairments given the insufficient body of literature. However, the Cochrane review did not look in detail at the type of training nor at the type of intervention outcomes (fundamental or clinical measures) that were studied. This last aspect is particularly important because skeletal muscle responses and adaptations to exercise and training do not limit themselves to maximal strength or endurance gains; many changes can occur at a physiological, functional or participation levels. A better understanding of the physiological responses and adaptations can also help uncover new intervention possibilities, such as a pharmaceutical agent combined with exercise or training to limit muscle impairments. Therefore, the main purpose of this scoping review is to map the existing literature relative to the effects of exercise and training undertaken to limit skeletal muscle impairments in DM1 and thereby identify gaps in the literature and informing where more research is needed. Cochrane systematic review methodology, which is more restrictive in their study selection, cannot be used to undertake this type of mapping of the literature.

\section{Methods}

In this review, the framework Methodology for JBI Scoping Reviews was used to develop the methods [26]. This framework is the best to use in this review because it has been designed to be broad enough to include any kind of existing scientific literature, thus allowing the 
most complete mapping on the desired subject and allowing to better achieve the objective of this study.

\section{Research question}

The scoping review aimed to answer the following question: "What is known about the effects of exercise and training that aim to reduce skeletal muscle impairments of patients with myotonic dystrophy type 1?" The question was built using the PCC (population, context and concept) model [26] where DM1 was the population, exercise or training were the context and muscle responses or adaptations (clinical or physiological) were the concept.

\section{Identifying relevant studies}

A systematic literature search was undertaken on February 16th, 2017 with no date limitations to identify all relevant studies. The search was organised into three main concepts and sub-concepts: concept 1-myotonic dystrophy, concept 2-clinical interventions (2a: exercise training, 2b: rehabilitation), and concept 3-muscle (see Supplemental digital content 1).

These three concepts allow the identification of appropriate keywords thesaurus and synonyms according to each database. Four databases were searched: Additional file 1: Medline (EBSCO), Pubmed, CINAHL (EBSCO) and EMBASE. Duplicates and articles that were not in French or in English were removed (119 articles were removed due to language). The search strategy was reviewed by a knowledge broker. A total of 704 articles were obtained (690 English and 14 French).

\section{Study selection}

Two independent reviewers (M-PR and MM) screened the article titles and abstracts according to the following inclusion and exclusion criteria. Inclusion: (1) humans with DM1 and (2) experimental protocol relying on exercise or training. Exclusion: (1) studies that do not evaluate skeletal muscle responses or adaptations (clinical or physiological), (2) reviews that covered articles that were already included in this scoping review and (3) pharmacological intervention at the same time of the exercise or training protocol. After the initial screening, the remaining articles were fully read and screened according to the same criteria by the same independent reviewers (M-PR and MM). In case of a disagreement, both reviewers discussed about the eligibility of the study. If a consensus was not reached, a third reviewer (ED) was consulted.

\section{Charting the data}

The data from the selected studies were then charted by one reviewer (M-PR) and reviewed by a second reviewer, a second-year physical therapy student with two years of experience as a research trainee in our lab, according to a data extraction grid to ensure standardized data extraction (Table 1). By working exclusively on projects focusing on DM1, both evaluators have some knowledge of the disease. Moreover, their training in physical therapy along with their experience as trainee in wet lab make them familiar with the continuum of outcome measures found in the literature. A beta version of the extraction grid was tested on three articles before the final grid was produced.

\section{Collating, summarizing and reporting the results}

The results were summarized under two main themes: outcome measures and exercise or training protocols. The outcome measures were subdivided in clinical measurements and physiological variables (top of Additional file 2). Clinical measurements were categorized as intervention outcomes (patient-reported outcome measures, functional tests, muscle endurance tests, aerobic capacity tests, muscle strength tests and other) and sub-categorized into specific measure types. Physiological variables were categorized as laboratory tool (myography, magnetic resonance imagery (MRI), muscle biopsy analysis and blood tests) and sub-categorized into studied physiological variables. Each of these sub-categories can be seen in Additional file 2. There were two types of exercise or training protocols: single session exercise and training programs. The type of training programs used (far left column of Additional file 2) were categorized as: neuromuscular electric stimulation (NMES), strength training, aerobic training, balance training and multiple rehabilitation interventions. Also, as the detailed protocol of some clinical measurements were not standardized, mostly

Table 1 Data extraction grid
Title/Journal
Author(s)
Year
Study eligibility
Country
Aims/Objectives
Diagnostic criteria, inclusion/exclusion criteria and phenotype
Number of DM1 participants and protocol compliance
Sex
Age
Other clinical manifestations of DM1 considered
Study design and exercise or training protocol (detailed)
Muscles targeted by the exercise or training protocol
Clinical measurements and detailed protocol
Physiological parameters evaluated
$P$ value, confidence interval
Results (detailed) 
concerning muscle endurance, aerobic capacity and strength testing, information needed to reproduce them were assessed and classified by two independent reviewers (M-PR and the second-year physical therapy student). If there was a disagreement, a third reviewer would decide (ED).

\section{Results}

The detailed steps of the systematic literature search can be found in the PRISMA flow chart (Fig. 1) [27]. A total of 704 papers were identified following the systematic search, from which 43 were selected based on title and abstract. These 43 papers were entirely read and both reviewers (M-PR and MM) excluded 22 papers according to inclusion and exclusion criteria (Fig. 1 for reasons of exclusion).

\section{Study characteristics}

Detailed information regarding the selected papers can be found in Table 2. Within the twenty-one selected studies, four were randomised controlled trials (RCT) [2, 28-30], twelve had before-after designs [3, 24, 31-40], two were cross over studies [41, 42], one was retrospective [43], one was a case study [44] and one was a case report [45]. Sample size varied between 1 and 35 participants depending on the design with most studies with before-after design or RCT with a sample size ranging from 10 to 35 DM1 participants.

\section{Exercise and training protocols}

(Left column of Additional file 2). Different exercise or training protocols have been used to study muscle responses or adaptations. As expected, single session exercises were done under supervision [31, 34, 36-40]. Regarding training programs, seven were done under healthcare professional supervision [24, 29, 33, 43-45], two presented a mixed supervision (professionally supervised and home-based sessions) [28, 41], one was done under parent's supervision [42] and four were performed at home, then without supervision [2, 3, 30, 32].

\section{Clinical measurements}

(Upper row - left of Additional file 2). Many standardized functional tests were used (Additional file 2), some studies used technology to assess function (Missaoui et al. 2010 [43]: a Statel stabilometer to assess balance and a locometer as a computer assisted movement analysis to evaluate gait) and homemade clinical functional tests such as measuring the time needed to do transfers (Lindeman et al. 1995 [30] and Conraads et al. 2002 [45]). Muscle endurance testing, strength testing, aerobic capacity and other clinical measurements are further detailed in Additional file 2. Many different clinical measurements were given throughout the selected studies and most of them relied on highly standardized protocols, however, this is not always the case. Therefore, the studies that included these types of measures were listed and reproducibility of their protocol was evaluated (Table 3).

\section{Physiological variables}

(Upper row - right of Additional file 2). Surface electromyography (SMEG) was the only method used to assess muscle electric activation within the included studies [2, 32, 34, 36, 40]. Esposito et al. 2017 [34] introduced mechano myography (MMG) to evaluate the degree of

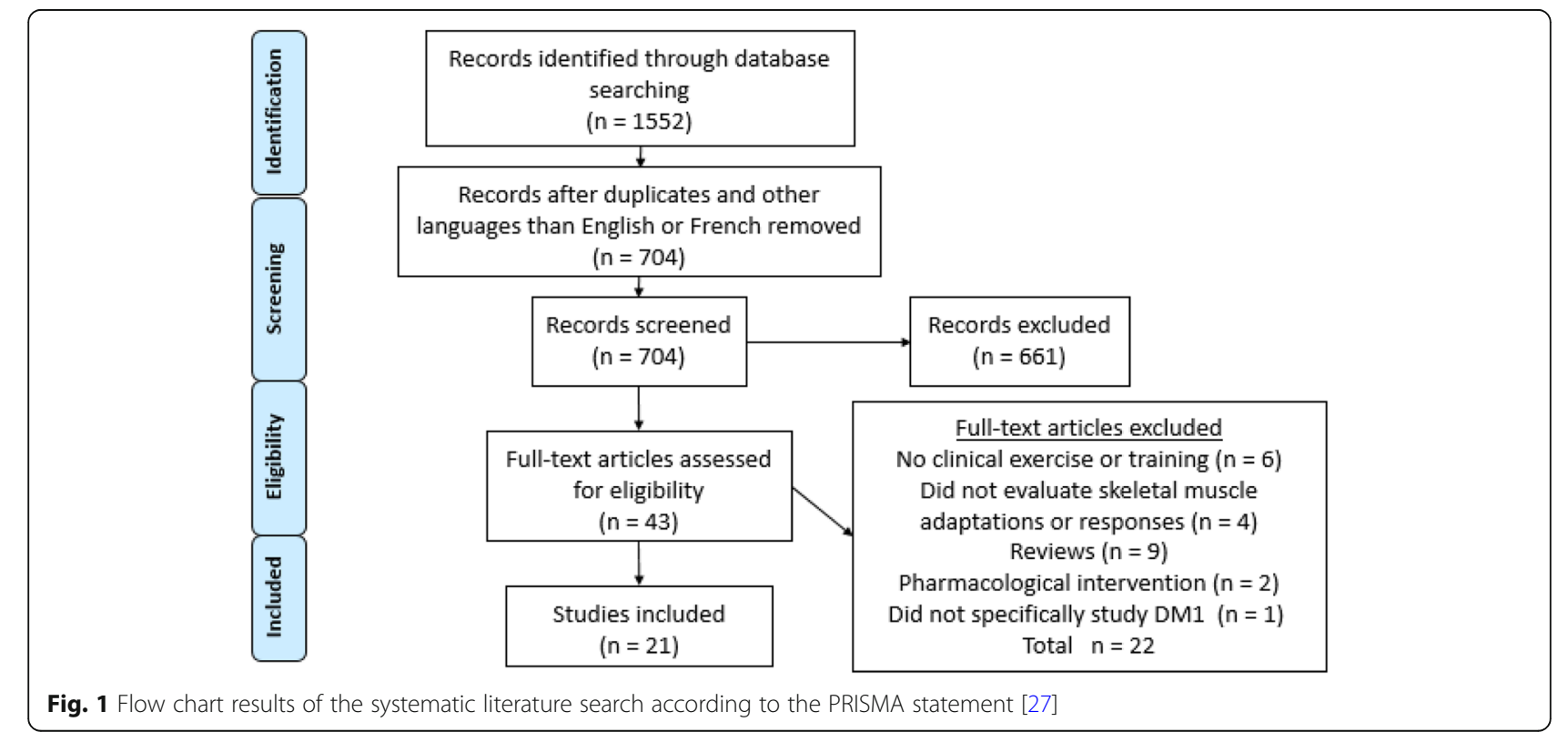




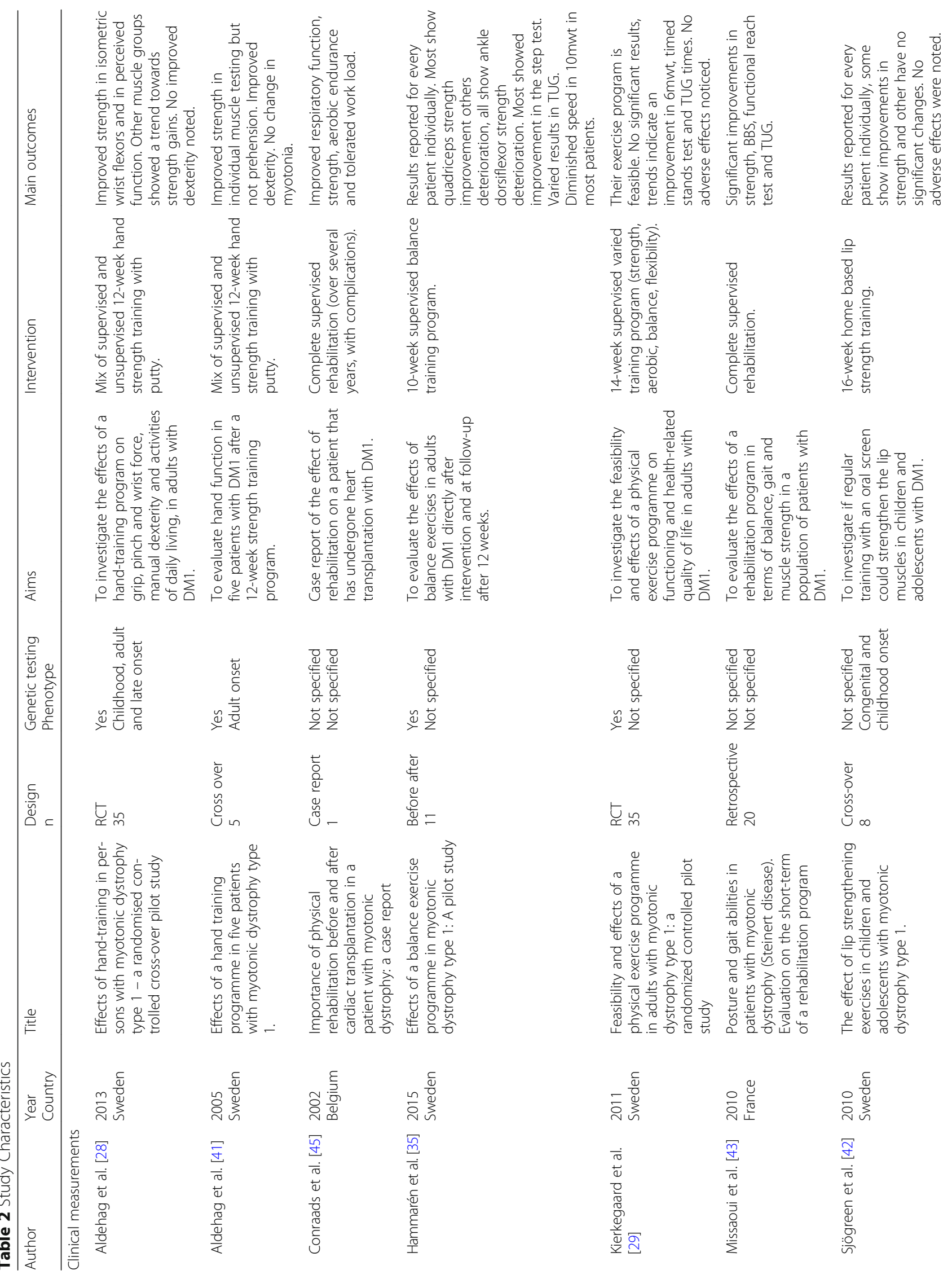




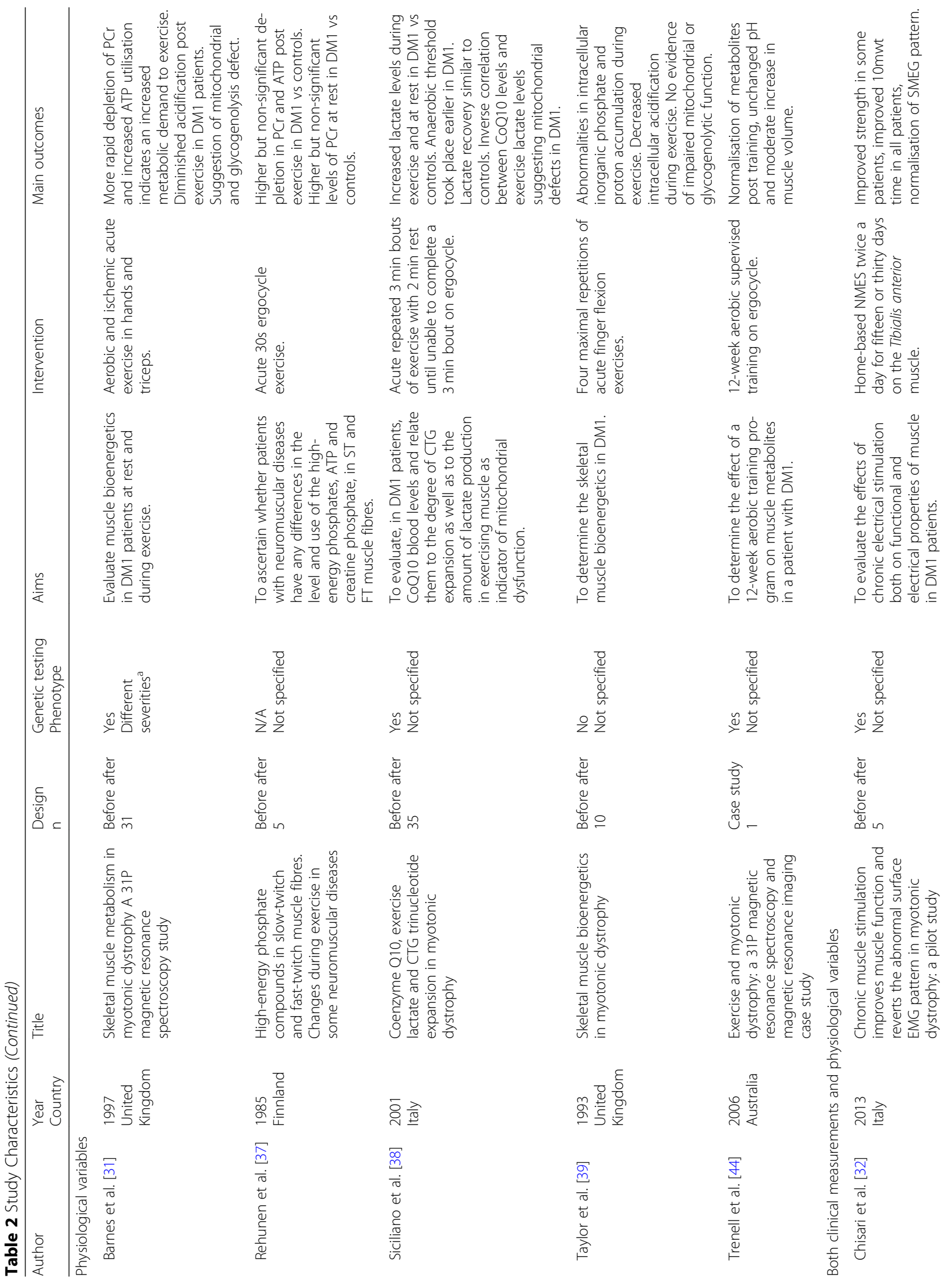




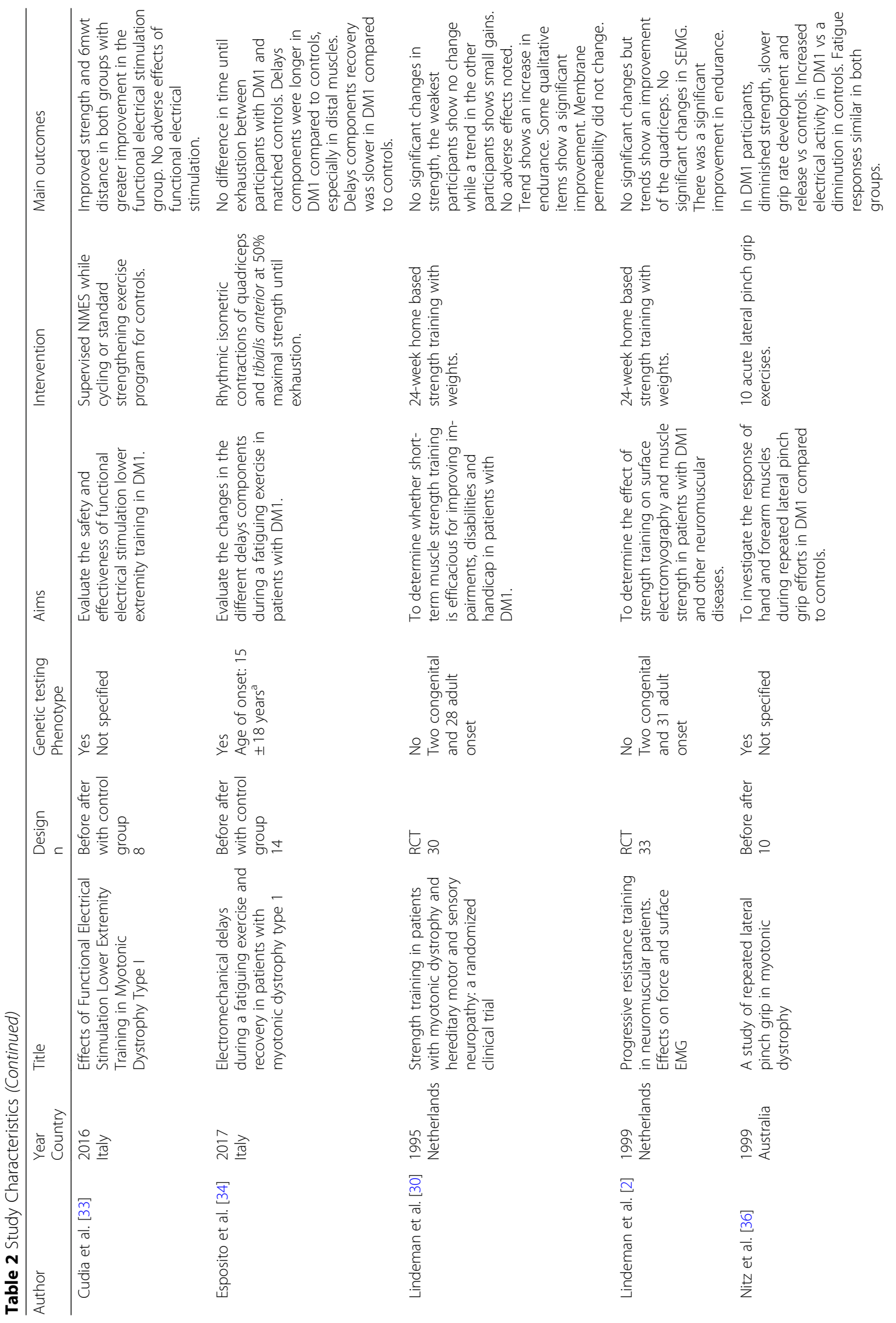




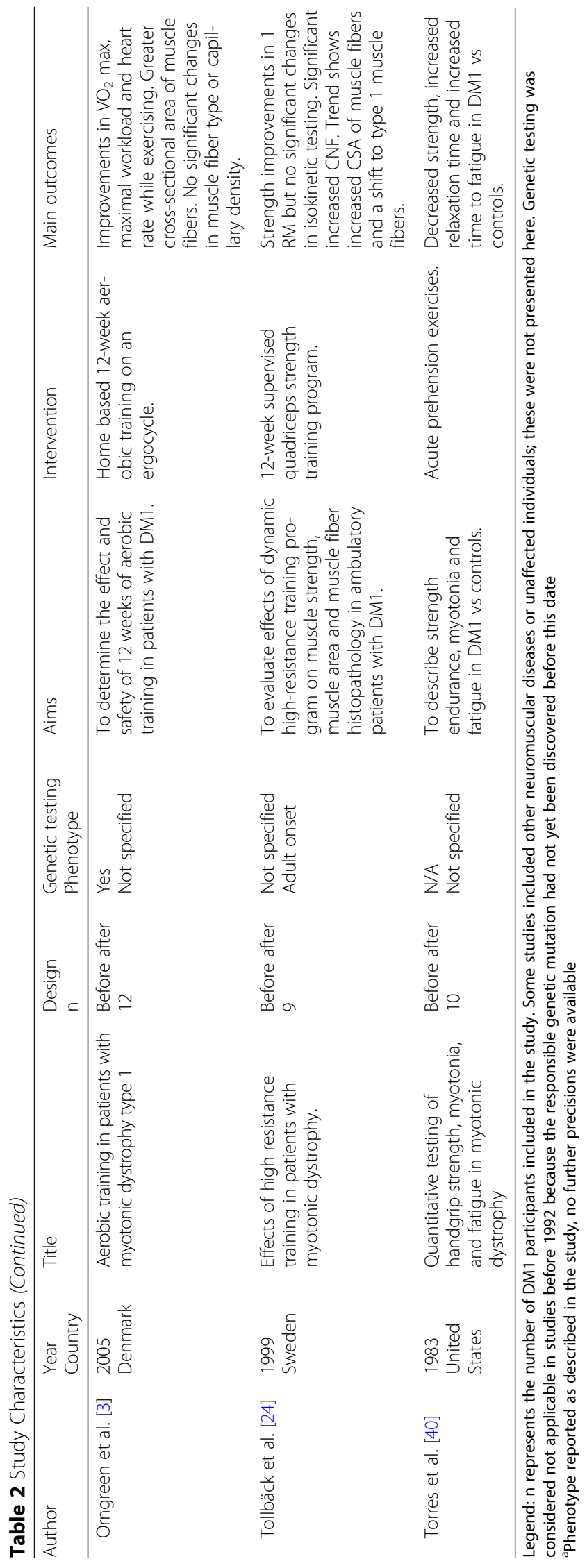


Table 3 Clinical measurements standardization

\begin{tabular}{|c|c|c|c|c|c|c|c|c|}
\hline & Muscle eno & ance & Aerobic capa & & Strengt & & & \\
\hline & $\begin{array}{l}\text { Time at } \\
50 \% \text { max } \\
\text { strength }\end{array}$ & $\begin{array}{l}\text { Time at } \\
80 \% \text { max } \\
\text { strength }\end{array}$ & $\begin{array}{l}\text { Submaximal } \\
\text { cycling and } \\
\text { walking }\end{array}$ & $\begin{array}{l}\mathrm{VO}_{2} \text { max (cycling), } \\
\text { heart rate and gas } \\
\text { exchanges }\end{array}$ & $1 \mathrm{RM}$ & $\begin{array}{l}\text { Isokinetic and } \\
\text { isometric computer } \\
\text { assisted device }\end{array}$ & MMT & $\begin{array}{l}\text { Force gauge } \\
\text { (handheld or } \\
\text { fixed) }\end{array}$ \\
\hline Aldehag 2005 [41] & & & & & & & & $\mathrm{O}$ \\
\hline Aldehag 2013 [28] & & & & & & & & $\mathrm{O}$ \\
\hline Chisari 2013 [32] & & & & & & & $\mathrm{O}$ & \\
\hline Conraads 2002 [45] & & & $x$ & & $x$ & $x$ & & \\
\hline Cudia 2016 [33] & & & & & & & $\mathrm{O}$ & \\
\hline Esposito 2017 [34] & $\mathrm{O}$ & & & & & & & $\mathrm{O}$ \\
\hline Hammarén 2015 [35] & & & & & & & & $\mathrm{O}$ \\
\hline Lindeman 1995 [30] & & $\mathrm{O}$ & & & & $\mathrm{O}$ & & \\
\hline Lindeman 1999 [2] & & $\mathrm{O}$ & & & & $x$ & & \\
\hline Missaoui 2010 [43] & & & & & & $x$ & & \\
\hline Nitz 1999 [36] & & & & & & & & $x$ \\
\hline Orngreen 2005 [3] & & & & $x$ & & & & \\
\hline Sjögreen 2010 [42] & $\mathrm{O}$ & & & & & & & $\mathrm{O}$ \\
\hline Tollbäck 1999 [24] & & & & & $\mathrm{O}$ & $\mathrm{O}$ & & \\
\hline Torres 1983 [40] & $\mathrm{O}$ & & & & & & & $\mathrm{O}$ \\
\hline
\end{tabular}

Legend: O: sufficient data for precise reproduction of protocol. X: insufficient data for precise reproduction of protocol

muscle mechanical activation. Blood tests were used by Siciliano et al. 2001 [38] to assess exercise-induced blood lactate levels as an indicator of mitochondrial function. Blood myoglobin levels were used in another study [30] to ensure that training did not induce negative effects. MRI was used to evaluate different muscular variables: 1) quadriceps muscle volume [24, 33, 44], 2) fatty infiltration of the tibialis anterior [33], 3) intramuscular $\mathrm{pH}$ [31, 39, 44] and 4) bioenergetics, such as adenosine tri-phosphate (ATP), inorganic phosphate or phosphocreatine (PCr) [31, 39, 44]. Muscle biopsy was used to assess muscle bioenergetics (ATP and PCr) in a study conducted by Rehunen et al. 1985 [37]. It was also used by Orngreen et al. 2005 [3] to determine capillary density. Then, fiber type was assessed in two different studies $[24,37]$ and muscle fiber cross sectional area (CSA) was also evaluated in two papers [3, 24]. The proportion of centrally nucleated fibers (CNF) was evaluated in one study [24].

\section{Outcome types}

Main outcome types of included studies can be found in Table 2. During the analysis of the effect of exercise or training on skeletal muscle in DM1, it appeared that the type of outcomes should be analyzed through two types of intervention, as their natures are completely different: muscular responses induced by acute exercise (single session) and muscular adaptations induced by training programs are thus presented separately.

\section{Single session exercise}

Regardless of the assessment method used, MRI or biopsy, all three studies measuring bioenergetics showed faster PCr and ATP depletion during exercise in DM1 patients in comparison to unaffected individuals [31, 37, 39]. One of these three papers showed an increased intramuscular $\mathrm{PCr}$ concentration at rest compared to unaffected individuals [37]. One of these papers along with one using blood tests to evaluate blood lactate levels suggested mitochondrial defects [31, 38], but Barnes et al. 1997 [31] supported that this mechanism was not the main reason why DM1 patients experienced muscle weakness and wasting. All two studies [31, 39] evaluating intramuscular $\mathrm{pH}$, reported higher $\mathrm{pH}$ post-exercise and a quicker return to normal levels of $\mathrm{pH}$ post-exercise. Strength testing, in all three studies, showed lower maximal strength $[34,36,40]$ and longer relaxation time compared to healthy controls [34, 36, 40], which are consistent with muscle weakness and myotonia experienced by patients with DM1. In one of two studies evaluating muscle endurance [40], a longer time before fatigue (evaluated by calculating the time that the patient is able to maintain a $50 \%$ maximal strength contraction) was reported in DM1 individuals in comparison to unaffected ones. In contrast, the other study evaluating muscle endurance, Esposito et al. 2017 [34] reported that rhythmic $6 \mathrm{~s}$ contractions at $50 \%$ of maximal strength led to the same time to exhaustion between DM1 and unaffected individuals. In the two studies evaluating root mean square (RMS) of electromyography (EMG) signal (an indicator of 
physiological electrical activity), one found RMS increased compared to unaffected individuals [36] and continued to increase with repetitions of exercise while the other found no significant differences of RMS between DM1 and control subjects during exercise [34].

\section{Training programs}

Positive effects of training programs on patient-reported outcomes, such as improved self-perception of occupational performance, are found in all five studies measuring this outcome $[3,28,30,35,41]$. Lindeman et al. 1999 [2] showed a significant increase in muscle endurance while Lindeman et al. 1995 [30] showed a trend towards increased muscle endurance. In addition, Sjögreen et al. [42] showed an increase in muscle endurance in some participants. These were the only three studies that evaluated the effect of a training program on muscle endurance. Aerobic capacity was improved in both studies that evaluated it $[3,45]$, with one study showing an improvement in respiratory function [45]. Improvement in functional tests was found in four studies of the ten studies that included them [32, 33, 41, 43]. All the other studies showed no change except for a decreased performance in the $10 \mathrm{~m}$ walk test $(10 \mathrm{mwt})$ that was reported by Hammarén et al. 2015 [35], in which they hypothesized it was due to a greater caution from the patients because they might be better aware of the risk of falling. Maximal strength improvement was reported in nine out of eleven studies that measured it $[24,28$, $32,33,35,41-43,45]$. It is to be noted that in some of these studies, strength improvement was not observed in all participants, in all muscle groups trained or in different assessment methods [24, 28, 32, 33, 35, 41, 42]. One study reported loss in maximal strength in some patients [35]. One study out of two showed an exercise-induced normalization of electromyographic patterns through SMEG evaluation [32]. Bioenergetics (ATP and PCr) were normalized without a change in abnormal $\mathrm{pH}$ in the only study that evaluated it following a training program [44]. One study out of three reported a slight increase in muscle volume through MRI [44]. The two studies that used muscle biopsies to assess muscle fiber CSA reported an increase, one only a trend in type I fibers [24] and one in both type I and type IIa [3]. These findings are encouraging because they support muscle growth. Fiber type and capillary density were not reported to change $[3,24]$. The only study evaluating these factors reported an increase in CNF [24] and unchanged blood myoglobin levels were also [30].

\section{Discussion}

The aim of this scoping review was to map out what is known about the effects of exercise or training program used to counteract muscle impairments in people affected by DM1 in order to guide future research. There is an obvious lack of studies available to fully comprehend muscular immediate responses and long-term adaptations to exercise or training and some domains have been insufficiently or have not yet been investigated (Fig. 2). More literature related to exercise or training could be particularly useful for healthcare professionals, such as physical therapists, who need to know the right type and dosage of exercise to prescribe to their patients to limit the progression of muscle impairments. Although the majority of studies report no adverse effects of exercise or training, there is no evidence supporting positive effects and data are insufficient to have a clear understanding of the optimal parameters to use with this population. A previous systematic review has also showed no adverse effects of strength training in people with DM1 [25].

Being a scoping review, the methodological quality of the different studies has not been assessed and this constitutes a limit to the interpretation of the outcomes reported in the different studies. Furthermore, as the exercise and training protocols and outcome measures were highly heterogenous, data pooling is not yet a feasible option. As shown in this scoping review, there is no sufficient similar high-quality evidences available to carry out a systematic review or a meta-analysis with a research question such as "What is known about the effects of exercise or training that aim to reduce skeletal muscle impairments of patients with myotonic dystrophy type 1?". One preferred design seems to be before/after or crossover designs, which may be better suited for studies where few participants are available [46].

Exercise and training protocols have been separated into two types to facilitate the analysis. Single session exercise studies allowed the understanding of the early muscle responses to exercise of people with DM1 in comparison to controls. On the other hand, training programs gave information about muscular adaptations. These can lead to a better understanding of the benefits of long-term training and thus help healthcare professionals to choose an appropriate rehabilitation approach to manage skeletal muscle impairments. Studies using single session exercise have used more physiological variables to assess efficacy while studies using training programs tended to use more clinical measurements. This is not surprising since a short (unique) stimulus is not expected to induce clinically significant changes. However, the inclusion of physiological variables in studies using training programs would have given an important insight of the cellular and molecular mechanisms supporting the changes in clinical outcomes. This scoping review has outlined that the focus in the physiological variables was on muscle conductivity, bioenergetics, mitochondrial activity, intramuscular $\mathrm{pH}$, centrally 


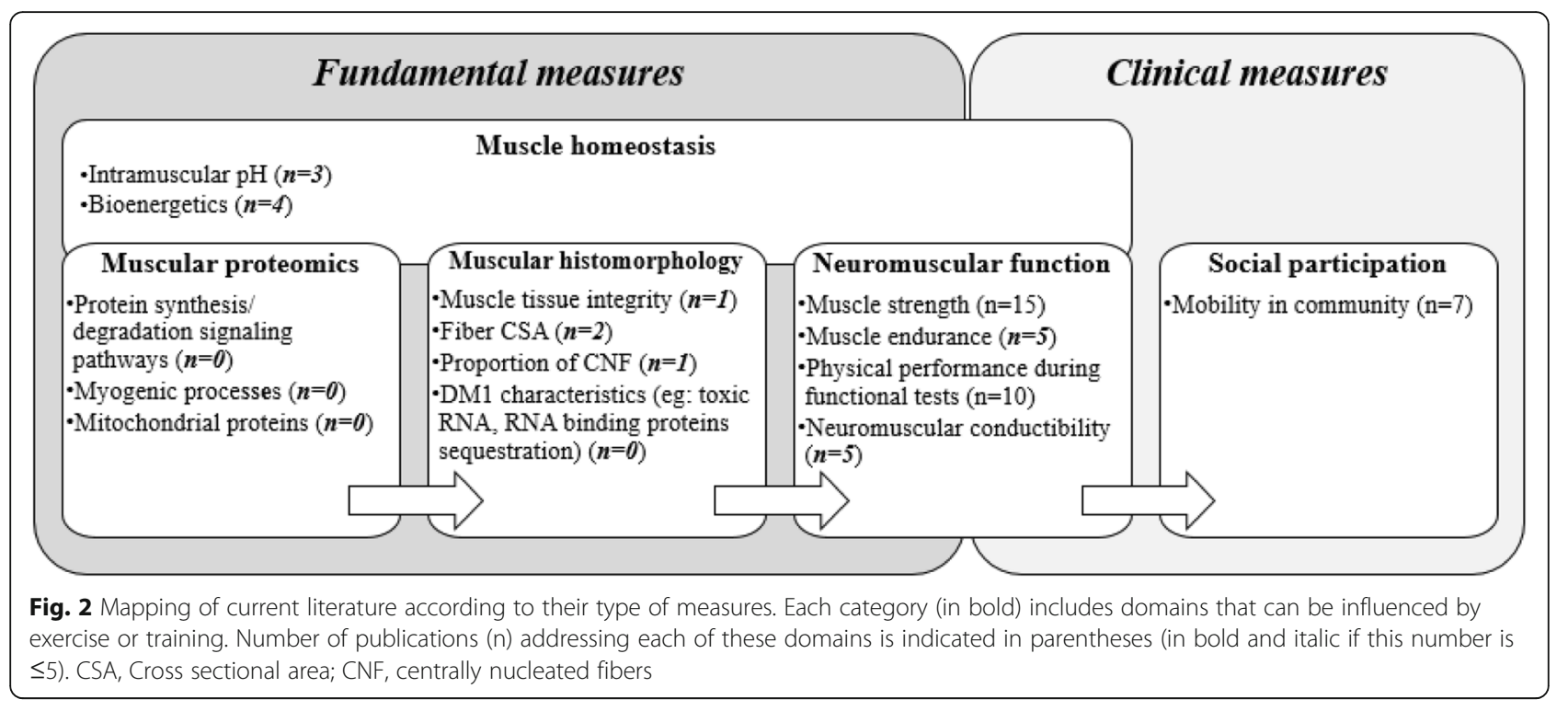

nucleated fibers and muscle fiber type and size. While it is known in DM1 that skeletal muscle has multiple alterations in inflammatory status, myogenesis and protein synthesis/degradation balance $[1,18-22]$, there are, to this day, no studies that consider the impact of exercise or training on these aspects. This knowledge would be a key component to help to understand why patients do not all show the same response to exercise or training throughout the different studies, thus leading to a better comprehension of the dosage and type of exercise or training that should be prescribed to obtain optimal results.

It is to be noted that at the time period when the systematic search was conducted, no animal studies about the effect of exercise in DM1 were found. This scoping review was thus directed on human studies only. It is only very recently (January 2019) that the first animal model study was published to assess the effect of exercise in DM1 (access to a running wheel for 7 weeks) by Manta et al. [47] This study demonstrates very interesting results about the effect of exercise in DM1: it improves strength and endurance, normalizes electromyographic signal (an indication of reduced myotonia), increases mitochondrial content, decreases accumulation of CUG RNA resulting in a decreased sequestration of MBNL-1 among other physiological benefits [47]. These results are consistent with some uncovered in this scoping review where exercise had beneficial effects on strength $[24,28,32,33,35$, 41-43, 45], endurance [2] or electromyographic signal [32]. However, many of the physiological outcomes measured by Manta et al. have not yet been measured in humans, showing another gap in current scientific literature.

From a clinical standpoint, this scoping review showed that there is a major lack in the body of evidence available to help clinicians to base their rehabilitation interventions on solid literature for their DM1 patients. In addition, compliance of patients to participate to a rehabilitation intervention therapy can be hampered by other symptoms since DM1 is a multisystemic disease [9]. For example, it has been shown that $40 \%$ of patients with DM1 suffer from apathy [48]. This can have a major impact on the reported outcome of exercise and training. Cardiac impairments are also frequently reported in DM1 [49], which can lead to restrictions in exercise and training participation. It is worth to be mentioned that these are only a few examples of other symptoms of DM1 that can interfere with exercise or training, which should be investigated in the future.

A lack in the standardization of some clinical measurements has also been observed between the selected studies. This element is particularly important to control with DM1 patients since many other confounding factors arising from the multisystemic nature of the disease may already influence the results. An example of this is strength testing, which represents one of the major outcomes used to assess muscle adaptations following a training program. The issue about lower limb strength assessment in DM1 patients has already been evaluated in further detail by a systematic review [50] where different methods were described and very few of them had sufficient methodological descriptions to allow protocol reproducibility. In addition, manual muscle testing has been shown to significantly lack sensibility and validity to adequately measure muscle strength in DM1 population [8]. In this scoping review, only two studies out of 15 that evaluate maximal strength used only MMT to assess strength; however, this still represents $9 \%$ of the accepted studies. Furthermore, these two studies were the only ones using a NMES training program, which 
greatly hampers the strength of the evidence regarding this type of training.

Another interesting point brought on by Petitclerc et al. 2017 is that adult and late-onset phenotypes should not be pooled together to assess muscle strength because of their different maximal strength loss profile over time [8]. Congenital, infantile, childhood onset phenotypes are already known to be even more clinically different from the adult and late-onset phenotypes [49]. This brings the hypothesis that some methodological risks are associated to the pooling of different phenotypes when evaluating the effectiveness of an intervention aimed at decreasing muscular impairments. In this scoping review, many studies mixed different phenotypes or do not even specify the phenotypes of their participants. Future studies should consider this important element when recruiting their participants. Moreover, many studies published after 1992 did not specify nor did genetic testing to validate if their participants were true DM1 patients. As the mechanisms underlying maximal muscle strength loss vary greatly between neuromuscular diseases, genetic testing should be a standard procedure in DM1 research.

\section{Conclusion}

This paper maps and summarizes all existent literature about the effect of exercise or training in humans with DM1. We also offer a clear framework to guide future studies. There is a great deal of research that still needs to be done in order to fully understand how skeletal muscles in DM1 patients respond to exercise and training. Many studies looked at neuromuscular function, however there are still an insufficient number of RCTs to come to clear conclusions. Furthermore, no studies have yet evaluated the effects of exercise or training on muscle proteomics, that are essential to understand the underlying mechanisms of the observed adaptations. Once a better understanding will be reached, clinicians will be able to prescribe interventions more effectively and thus better manage muscle wasting and weakness.

\section{Additional file}

Additional file 1: Search words and headings. All search words and headings used in the different data bases (Medline (EBSCO), Pubmed, CINAHL (EBSCO) and EMBASE) can be found in this additional file. (DOCX $19 \mathrm{~kb}$ )

Additional file 2: Summary of measurements, parameters and exercise or training protocols. (PDF $162 \mathrm{~kb}$ )

\section{Abbreviations}

10mwt: 10-m walk test; 1RM: One maximal repetition test; 6mwt: 6-min walk test; ATP: Adenosine triphosphate; BBS: Berg balance scale; CNF: Centrally nucleated fibers; CoQ10: Coenzyme Q10; CSA: Cross sectional area; CTG: Cytosine, thymine and guanine nucleotide triplet; DM1: Myotonic dystrophy type 1; DMPK: Dystrophy Myotonic Protein Kinase gene;
EMG: Electromyography; GSK3ß: Glycogen synthase kinase 3 beta; MMG: Mechanomyography; MMT: Manual muscle testing; MRI: Magnetic resonance imaging; NMES: Neuromuscular electrical stimulation; PCr: Phosphocreatine; RCT: Randomized controlled trial; RNA: Ribonucleic acid; SMEG: Surface electromyography; TUG: Timed up and go; $\mathrm{VO}_{2}$ max: Maximal volume of oxygen

\section{Acknowledgements}

The authors are pleased to acknowledge their partners in the completion of this manuscript. Annie Plourde, Ph.D., worked as a knowledge broker and helped throughout multiple steps of the completion of the manuscript: revision of the database search strategy and revision of the manuscript. Olivier Houle, a physical therapy student, reviewed the charting of the data and participated in the clinical protocol reproductivity evaluation.

\section{Funding}

The first author of this review (M-PR) received multiple scholarships to complete her master's degree from different organisms: Fonds de recherche du Québec - Santé; Canadian Institutes of Health Research; Fondation du Grand défi Pierre Lavoie and Corporation de recherche et d'action sur les maladies héréditaires. The last author (ED) also received funding from the Fondation de l'Université du Québec à Chicoutimi to hire an independent reviewer and a knowledge broker. CG holds a career-grant funding from Fonds de recherche en santé du Québec [grant no. 31011]. None of these funding bodies had any role in: the design of the study; the collection, analysis or interpretation of data; or writing the manuscript.

\section{Availability of data and materials}

The datasets used and/or analysed during the current study are available from the corresponding author on reasonable request.

\section{Authors' contributions}

$M-P R$ and ED participated in the conception and writing of the manuscript. M-PR and MM have participated in data collection and analysis. ED and CG have both critically revised the manuscript. All authors have read and approved the manuscript.

Ethics approval and consent to participate

Not applicable.

\section{Consent for publication}

Not applicable.

\section{Competing interests}

The authors declare that they have no competing interests.

\section{Publisher's Note}

Springer Nature remains neutral with regard to jurisdictional claims in published maps and institutional affiliations.

\section{Author details}

${ }^{1}$ Département des sciences de la santé, physiothérapie, Université du Québec à Chicoutimi, 555, boulevard de I'Université, Chicoutimi, Quebec G7H 2B1, Canada. ${ }^{2}$ Groupe de recherche interdisciplinaire sur les maladies neuromusculaires, Centre intégré universitaire de santé et de services sociaux du Saguenay-Lac-St-Jean, 2230 rue de I'Hôpital, Saguenay, Québec, Canada. ${ }^{3}$ Centre de recherche Charles-Le Moyne - Saguenay-Lac-Saint-Jean sur les innovations en santé, 2230 rue de l'Hôpital, Saguenay, Québec, Canada., Longueuil, Québec, Canada. ${ }^{4}$ Faculté de médecine et des sciences de la santé, Université de Sherbrooke, 3001, 12e Avenue Nord, Sherbrooke, Québec, Canada.

Received: 8 August 2018 Accepted: 6 February 2019

Published online: 05 March 2019

\section{References}

1. Halliday D, Ford GC, Edwards RH, Rennie MJ, Griggs RC. In vivo estimation of muscle protein synthesis in myotonic dystrophy. Ann Neurol. 1985;17:65-9. 
2. Lindeman E, Spaans F, Reulen J, Leffers P, Drukker J. Progressive resistance training in neuromuscular patients. Effects on force and surface EMG. J Electromyogr Kinesiol. 1999;9:379-84.

3. Orngreen MC, Olsen DB, Vissing J. Aerobic training in patients with myotonic dystrophy type 1. Ann Neurol. 2005;57:754-7.

4. Fu YH, Pizzuti A, Fenwick RG Jr, King J, Rajnarayan S, Dunne PW, Dubel J Nasser GA, Ashizawa T, de Jong P, et al. An unstable triplet repeat in a gene related to myotonic muscular dystrophy. Science. 1992;255:1256-8.

5. De Antonio M, Dogan C, Hamroun D, Mati M, Zerrouki S, Eymard B, Katsahian S, Bassez G. French myotonic dystrophy clinical N Unravelling the myotonic dystrophy type 1 clinical spectrum: A systematic registry-based study with implications for disease classification. Rev Neurol (Paris). 2016;172:572-80.

6. Gagnon C, Petitclerc E, Kierkegaard M, Mathieu J, Duchesne E, Hebert LJ. A 9-year follow-up study of quantitative muscle strength changes in myotonic dystrophy type 1. J Neurol. 2018;265(7):1698-705.

7. Mathieu J, Boivin H, Meunier D, Gaudreault M, Begin P. Assessment of a disease-specific muscular impairment rating scale in myotonic dystrophy. Neurology. 2001;56:336-40.

8. Petitclerc E, Hebert L, Mathieu J, Desrosiers J, Gagnon C. Lower limb muscle strength impairment in late-onset and adult myotonic dystrophy type 1 phenotypes. Muscle Nerve. 2017;56:57-63.

9. Gagnon C, Mathieu J, Jean S, Laberge L, Perron M, Veillette S, Richer L, Noreau L. Predictors of disrupted social participation in myotonic dystrophy type 1. Arch Phys Med Rehabil. 2008;89:1246-55.

10. Mateos-Aierdi AJ, Goicoechea M, Aiastui A, Fernandez-Torron R, Garcia-Puga M, Matheu A, Lopez de Munain A. Muscle wasting in myotonic dystrophies: a model of premature aging. Front Aging Neurosci. 2015;7:125.

11. Meola G. Clinical aspects, molecular pathomechanisms and management of myotonic dystrophies. Acta Myol. 2013;32:154-65.

12. Jones K, Wei C, lakova P, Bugiardini E, Schneider-Gold C, Meola G, Woodgett J, Killian J, Timchenko NA, Timchenko LT. GSK3beta mediates muscle pathology in myotonic dystrophy. J Clin Invest. 2012;122:4461-72.

13. Bhagavati $S$, Shafiq $S A, X u W$. (CTG) $n$ repeats markedly inhibit differentiation of the C2C12 myoblast cell line: implications for congenital myotonic dystrophy. Biochim Biophys Acta. 1999;1453:221-9.

14. Bigot A, Klein AF, Gasnier E, Jacquemin V, Ravassard P, Butler-Browne G, Mouly V, Furling D. Large CTG repeats trigger p16-dependent premature senescence in myotonic dystrophy type 1 muscle precursor cells. Am J Pathol. 2009;174:1435-42.

15. Furling D, Coiffier L, Mouly V, Barbet JP, St Guily JL, Taneja K, Gourdon G, Junien C, Butler-Browne GS. Defective satellite cells in congenital myotonic dystrophy. Hum Mol Genet. 2001;10:2079-87.

16. Furling D, Lemieux D, Taneja K, Puymirat J. Decreased levels of myotonic dystrophy protein kinase (DMPK) and delayed differentiation in human myotonic dystrophy myoblasts. Neuromuscul Disord. 2001;11:728-35.

17. Sabourin LA, Tamai K, Narang MA, Korneluk RG. Overexpression of 3'untranslated region of the myotonic dystrophy kinase CDNA inhibits myoblast differentiation in vitro. J Biol Chem. 1997;272:29626-35.

18. Griggs RC, Halliday D, Kingston W, Moxley RT 3rd. Effect of testosterone on muscle protein synthesis in myotonic dystrophy. Ann Neurol. 1986;20:590-6.

19. Nadaj-Pakleza A, Lusakowska A, Sulek-Piatkowska A, Krysa W, Rajkiewicz M, Kwiecinski H, Kaminska A. Muscle pathology in myotonic dystrophy: light and electron microscopic investigation in eighteen patients. Folia Morphol (Warsz). 2011:70:121-9.

20. Tohgi H, Utsugisawa K, Kawamorita A, Yamagata M, Saitoh K, Hashimoto K. Effects of CTG trinucleotide repeat expansion in leukocytes on quantitative muscle histopathology in myotonic dystrophy. Muscle Nerve. 1997;20:232-4.

21. Fernandez-Real JM, Molina A, Broch M, Ricart W, Gutierrez C, Casamitjana R, Vendrell J, Soler J, Gomez-Saez JM. Tumor necrosis factor system activity is associated with insulin resistance and dyslipidemia in myotonic dystrophy. Diabetes. 1999:48:1108-12.

22. Zhang L, Lee JE, Wilusz J, Wilusz CJ. The RNA-binding protein CUGBP1 regulates stability of tumor necrosis factor mRNA in muscle cells: implications for myotonic dystrophy. J Biol Chem. 2008;283:22457-63.

23. Ravel-Chapuis A, Belanger G, Cote J, Michel RN, Jasmin BJ. Misregulation of calcium-handling proteins promotes hyperactivation of calcineurin-NFAT signaling in skeletal muscle of DM1 mice. Hum Mol Genet. 2017;26:2192-206.

24. Tollbäck A, Eriksson S, Wredenberg A, Jenner G, Vargas R, Borg K, Ansved T. Effects of high resistance training in patients with myotonic dystrophy. Scand J Rehabil Med. 1999;31:9-16.
25. Voet NB, van der Kooi EL, Riphagen II, Lindeman E, van Engelen BG, Geurts AC. Strength training and aerobic exercise training for muscle disease. Cochrane Database Syst Rev. 2013;CD003907.

26. The Joanna Briggs Institute. The Joanna Briggs institute Reviewers' manual 2015: methodology for JBI scoping reviews. Adelaide: The Joanna Briggs Institute; 2015

27. Moher D, Liberati A, Tetzlaff J, Altman DG, Group P. Preferred reporting items for systematic reviews and meta-analyses: the PRISMA statement. BMJ 2009;339:b2535

28. Aldehag A, Jonsson H, Lindblad J, Kottorp A, Ansved T, Kierkegaard M. Effects of hand-training in persons with myotonic dystrophy type 1--a randomised controlled cross-over pilot study. Disabil Rehabil. 2013;35: 1798-807.

29. Kierkegaard M, Harms-Ringdahl K, Edström L, Widén Holmqvist L, Tollbäck A. Feasibility and effects of a physical exercise programme in adults with myotonic dystrophy type 1: a randomized controlled pilot study. J Rehabil Med. 2011:43:695-702.

30. Lindeman E, Leffers P, Spaans F, Drukker J, Reulen J, Kerckhoffs M, Koke A. Strength training in patients with myotonic dystrophy and hereditary motor and sensory neuropathy: a randomized clinical trial. Arch Phys Med Rehabil. 1995;76:612-20.

31. Barnes PR, Kemp GJ, Taylor DJ, Radda GK. Skeletal muscle metabolism in myotonic dystrophy a 31P magnetic resonance spectroscopy study. Brain. 1997;120(Pt 10):1699-711.

32. Chisari C, Bertolucci F, Dalise S, Rossi B. Chronic muscle stimulation improves muscle function and reverts the abnormal surface EMG pattern in myotonic dystrophy: a pilot study. J Neuroeng Rehabil. 2013;10:94.

33. Cudia P, Weis L, Baba A, Kiper P, Marcante A, Rossi S, Angelini C, Piccione F. Effects of functional electrical stimulation lower extremity training in myotonic dystrophy type I...A pilot controlled study. Am J Phys Med Rehab. 2016;95:809-17.

34. Esposito F, Ce E, Rampichini S, Monti E, Limonta E, Fossati B, Meola G. Electromechanical delays during a fatiguing exercise and recovery in patients with myotonic dystrophy type 1. Eur J Appl Physiol. 2017;117(3): $551-66$.

35. Hammarén $\mathrm{E}$, Lindberg C, Kjellby-Wendt G. Effects of a balance exercise programme in myotonic dystrophy type 1: a pilot study. Eur J Phys. 2015;17: 123-31.

36. Nitz J, Burns Y, Wuthapanich N, Jackson R. A study of repeated lateral pinch grip in myotonic dystrophy. Physiother Res Int. 1999:4:1-11.

37. Rehunen S, Karli P, Härkönen M. High-energy phosphate compounds in slow-twitch and fast-twitch muscle fibres. Changes during exercise in some neuromuscular diseases. J Neurol Sci. 1985;67:299-306.

38. Siciliano G, Mancuso M, Tedeschi D, Manca ML, Renna MR, Lombardi V, Rocchi A, Martelli F, Murri L. Coenzyme Q10, exercise lactate and CTG trinucleotide expansion in myotonic dystrophy. Brain Res Bull. 2001;56: 405-10.

39. Taylor DJ, Kemp GJ, Woods CG, Edwards JH, Radda GK. Skeletal muscle bioenergetics in myotonic dystrophy. J Neurol Sci. 1993;116:193-200.

40. Torres C, Moxley RT, Griggs RC. Quantitative testing of handgrip strength, myotonia, and fatigue in myotonic dystrophy. J Neurol Sci. 1983;60:157-68.

41. Aldehag AS, Jonsson $H$, Ansved T. Effects of a hand training programme in five patients with myotonic dystrophy type 1. Occup Ther Int. 2005;12:14-27.

42. Sjögreen $L$, Tulinius $M$, Kiliaridis $S$, Lohmander $A$. The effect of lip strengthening exercises in children and adolescents with myotonic dystrophy type 1. Int J Pediatr Otorhinolaryngol. 2010;74:1126-34.

43. Missaoui B, Rakotovao E, Bendaya S, Mane M, Pichon B, Faucher M, Thoumie P. Posture and gait abilities in patients with myotonic dystrophy (Steinert disease). Evaluation on the short-term of a rehabilitation program. Ann Phys Rehabil Med. 2010;53:387-98.

44. Trenell Ml, Thompson CH, Sue CM. Exercise and myotonic dystrophy: a 31P magnetic resonance spectroscopy and magnetic resonance imaging case study. Ann Neurol. 2006;59:871-2

45. Conraads VM, Beckers PJ, Vorlat A, Vrints CJ. Importance of physical rehabilitation before and after cardiac transplantation in a patient with myotonic dystrophy: a case report. Arch Phys Med Rehabil. 2002;83:724-6.

46. Griggs RC, Batshaw M, Dunkle M, Gopal-Srivastava R, Kaye E, Krischer J, Nguyen T, Paulus K, Merkel PA, rare diseases clinical research N. Clinical research for rare disease: opportunities, challenges, and solutions. Mol Genet Metab. 2009;96:20-6. 
47. Manta A, Stouth DW, Xhuti D, Chi L, Rebalka IA, Kalmar JM, Hawke TJ, Ljubicic V. Chronic exercise mitigates disease mechanisms and improves muscle function in myotonic dystrophy type 1 mice. J Physiol. 2019. https:// doi.org/10.1113/JP277123.

48. Gallais B, Montreuil M, Gargiulo M, Eymard B, Gagnon C, Laberge L. Prevalence and correlates of apathy in myotonic dystrophy type 1. BMC Neurol. 2015;15:148.

49. Bird TD. Myotonic Dystrophy Type 1. In: Pagon RA, Adam MP, Ardinger HH, Wallace SE, Amemiya A, LJH B, Bird TD, Ledbetter N, Mefford HC, RJH S, Stephens K, editors. GeneReviews [Internet]. Seattle: University of Washington; 1993-2019.

50. Petitclerc E, Hebert L, Desrosiers J, Gagnon C. Lower limb muscle impairment in myotonic dystrophy type 1: the need for better guidelines. Muscle Nerve. 2015;51:473-8.

Ready to submit your research? Choose BMC and benefit from:

- fast, convenient online submission

- thorough peer review by experienced researchers in your field

- rapid publication on acceptance

- support for research data, including large and complex data types

- gold Open Access which fosters wider collaboration and increased citations

- maximum visibility for your research: over $100 \mathrm{M}$ website views per year

At $\mathrm{BMC}$, research is always in progress.

Learn more biomedcentral.com/submissions 